\title{
Conference Scene
}

Immunotherapy reaches new milestones in cancer eradication

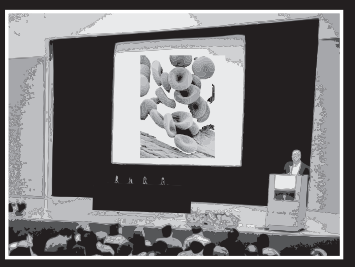

\section{Highlights of the 2nd International Cancer Immunotherapy and Immunomonitoring (CITIM) Conference} 2-5 May 2011, Budapest, Hungary

Biotherapy is widely considered as the fourth treatment modality for patients with cancer, and uses the constantly increasing knowledge in molecular biology, cell biology and immunology. Biotherapy uses naturally occurring biological molecules (e.g., cytokines and antibodies) or works by the manipulation of normal biological mechanisms (controlling or inhibiting tumor growth). Important achievements in anticancer drug development are immunotherapeutic strategies recently approved by the US FDA as well as clinical data of the cancer patients treated in clinical trials. There is a need to expand these novel cancer immunotherapeutic modalities for cancer patients all over the world. To meet that goal, it is essential to spread the information, to summarize the new clinical data and to draw the conclusions from the clinical and preclinical investigations. These frontline tasks can be well advanced by organizing international conferences in this domain in less scientifically developed countries, with a significant tumor burden statistics. Therefore, special efforts were done to organize the $2 \mathrm{nd}$ International Cancer Immunotherapy and Immunomonitoring Conference (CITIM-2011) in Hungary.

\section{Immunotherapy holds promise}

The last decade's progress in tumor immunology has improved the understanding of the role of host immune system in cancer development, but the whole field is far from being completely understood. With the new technologies and improved molecular methods, potentially immunogenic tumor antigens could be identified, which can served as suitable targets for immune effector cells in innovative immunotherapeutic strategies, as well as novel biomarkers for early cancer diagnostic. High-throughput gene-expression analysis has opened new opportunities for revealing key mechanisms of immunemediated tumor rejection [1-3]. The variety of immunotherapeutic approaches to cancer treatment grows steadily, from nonspecific immunotherapy to antigen-specific modalities, such as adoptive T-cell transfer and dendritic cell (DC)-based vaccines. The steadfast development of molecular immunological and biotechnological techniques, as well as genetic engineering and transfection approaches, resulted in new strategies such as chimeric antigen receptor (CAR)-modified T-cell technology that has already been successfully introduced into the clinic [4]. Here, we highlight some new and unique data that were presented at Cancer Immunotherapy and Immunomonitoring Conference (CITIM) conference. It became evident that all presentations at CITIM-2011 conference provided additional answers for cancer immunological questions and might be developed into effective anticancer therapeutic approach in the future.

\section{Main conference goals reached}

Hot-topic keynote and plenary lectures, as well as selected talks and numerous poster presentations provided a considerable amount of new ideas in tumor immunology, cancer diagnostics and therapies, which can advance medical and biomedical immunological sciences and clinical practice. We focus here on selected topics since there are also other conference papers to be published [5,6]. Exploitation of immune mechanisms and evasion of immune surveillance are properties that cancer cells acquire during tumor development. Comprehensive cellular, molecular and genetic investigations have lead to the evidence of immune hallmarks of cancer: chronically inflamed microenvironment, evaded immune recognition and suppression of immune reactivity, among others [7]. Taking into consideration the immune hallmarks of cancer and the possible countermeasures, doors open to widespread cancer immunoprevention and to innovative and more efficacious cancer immunotherapies. Novel concepts and strategies have been outlined to identify, monitor and predict efficacy of cancer immunotherapy protocols. CITIM
Beatrix Kotlan ${ }^{\dagger 1}$, Viktor Umansky ${ }^{2}$, Anatoli M Malyguine ${ }^{3}$,

Francesco M Marincola ${ }^{4}$

\section{\& Michael R Shurin ${ }^{5}$}

${ }^{1}$ Center of Surgical \& Molecular Tumor pathology, Department of Molecular Immunology \& Toxicology, National Institute of Oncology, 1122 Rath Gy Street 7-9, Budapest, Hungary

${ }^{2}$ Clinical Cooperation Unit, Dermato-Oncology, German Cancer Research Center, Heidelberg, 69 120, Germany ${ }^{3}$ Laboratory of Cell Mediated Immunity. SAIC-Frederick, Inc., NCl-Frederick, Frederick, MD 21 702, USA

${ }^{4}$ Infectious Disease \& Immunogenetics Section (IDIS), Departments of Translation Medicine, Clinical Center \& Center for Human Immunology (CHI), National Institute of Health, Bethesda, MD 20 892, USA ${ }^{5}$ Departments of Pathology \& Immunology, University of Pittsburgh Medical Center, Pittsburgh, PA 15 213, USA ${ }^{+}$Author for correspondence: Tel.: +361 2248600 ext. 2239 kotlanb@netscape.net 
conference attendees received a nice interpretation of current hot topic: 'Immunity and the malignant process'. From other presentations we have learnt about various novelties in cancer immunotherapeutic approaches following the previous international meetings in the field [8-10].

\section{Cancer has its questions \& answers: an asset for therapeutic approaches}

As an example, we heard comprehensive details about myeloid-derived suppressor cells (MDSCs) representing a key factor in the tumor escape processes. These immature cells of myeloid origin accumulate in tumor-bearing hosts, suppress immune responses, T-cell proliferation and cytokine production. Dmitry Gabrilovich (H Lee Moffitt Cancer Center, Tampa, FL, USA) has explained how the therapeutic targeting of MDSC can be reached in different ways, for example, using the differentiation agents, chemotherapy, tyrosine kinase inhibitor sunitinib, COX-2 inhibitors, among others. Susanne Ostrand-Rosenberg (Baltimore, MD, USA) gave further overview of the intensive investigations of the tumor microenvironment in terms of immune suppression mechanisms and the definition of multiple factors important in tumor progression. These studies help to overcome protumorigenic immune suppression that is a major obstacle to effective immunotherapy. Immunosuppressive pathways interfering with the antitumor immunity in cancer patients represent an area where molecular mechanisms, clinical relevance and suitability are still needed to be revealed. These and further ideas were discussed by Licia Rivoltini (Istituto Nazionale Tumori, Milan, Italy). We have learnt from Isaac P Witz (Tel Aviv University, Israel) how the nontumor cells in the tumor microenvironment, including lymphocytes, macrophages, fibroblasts and endothelial cells, shape the malignant phenotype of the tumor and its dissemination. The interactions of the tumor cells with the microenvironment of the specific organ sites regulate the site-specific metastases to these organs. Human-to-mouse xenograft models for melanoma and neuroblastoma help to establish a molecular signature of site-specific metastasis, identify cancer genes controlled by sitespecific microenvironment and identify candidates for site-specific therapeutic targets. Numerous molecules involved in the tumor-stroma interactions might also serve as specific biomarkers or therapy targets. Defining inflammation and the presence of inflammatory cells in the tumor is another critical aspect of cancer pathobiology. Ron Apte (Ben-Gurion University, Beer-Sheva, Israel) pointed out that IL-1 is a major molecule that tilts the balance between destructive inflammation and activation of antitumor immunity, and that IL- $1 \alpha$ and IL-1 $\beta$ differ dramatically in their effect on the malignant process. A talk of Zoltan Szekanecz (University of Debrecen, Hungary) was focused on the common targets of anticancer and anti-inflammatory therapy such as anticytokine agents, inhibitors of chemokines, adhesion molecules, T-cell costimulation and small molecules. There are interesting pathogenetic crossroads that link rheumatic diseases with cancer (e.g., angiogenesis inhibitors used in oncology have been introduced to arthritis trials). An intrinsic pathway of inflammation driven in tumor cells and an extrinsic pathway driven by chronic inflammatory conditions could be identified [11,12]. Novel results of Galina Shurin (University of Pittsburgh, PA, USA) demonstrated that accumulation of immune regulatory cells, such as MDSCs, regulatory DCs and regulatory $\mathrm{T}$ cells, is cancer and tissue specific and that different myeloid regulators might play different roles in tumor progression and inhibition of the antitumor immunity. Recent findings of Michael Shurin (University of Pittsburgh, PA, USA) proved for the first time that certain antineoplastic chemotherapeutic agents directly upregulate development, maturation and functional activation of conventional DCs (cDCs) in vitro and in vivo if used in ultralow noncytotoxic concentrations. This unexpected phenomenon was termed chemomodulation. Ultralow-dose nontoxic chemomodulation not only directly activated DCs, but also decreased tumor-induced suppression of $\mathrm{cDC}$ in the tumor microenvironment and could convert tumor-induced regulatory DCs into immunostimulatory cDC subsets. Thus, chemomodulation 
of tumor environment may improve the efficacy of modern immunotherapeutic approaches for cancer. Viktor Umansky (German Cancer Research Center, Heidelberg, Germany) presented data on chronic inflammation and MDSC expansion and activation inducing immunosuppression in RET-transgenic spontaneous tumor model and suggested that the neutralization of MDSC immunosuppressive functions could be a key component of effective human melanoma immunotherapies. For instance, treatment of tumor-bearing mice with phosphodiesterase inhibitor or chemomodulation with ultralow-dose paclitaxel resulted in a marked delay of tumor progression and in a significant increase in animal survival. These effects were directly associated with a reduction in MDSC accumulation and function and increase in $\mathrm{CD}^{+} \mathrm{T}$ cells infiltrating skin melanoma and metastatic lymph nodes.

Human tumors produce different immunosuppressive factors, which by inhibiting the antitumor immune responses promote tumor progression. Theresa Whiteside (University of Pittsburgh Cancer Institute) focused on new pharmacologic blockade mechanisms related to the adenosinergic pathway that relieve suppression and restore immune responses. Anatoli Malyguine (NCI-Frederick, MD, USA) successfully developed and selected innovative ex vivo immunomonitoring methods and techniques that provide the most appropriate immune reactivity measures in determining the correlations between clinical and immunologic responses to specific immunotherapy in cancer patients. Paul Lehmann (Cellular Technology Ltd,
Shaker Heights, OH, USA) as the main conference sponsor being the founder of Cellular Technology Ltd in the USA and Europe, explained how to monitor both cytotoxic T-lymphocyte (CTL) frequency and functions with ELISPOT assays that gained increasing popularity for monitoring clinical trials worldwide.

One promising approach to cancer therapy is the induction of tumor-specific responses that selectively target the cancer. Straightforward steps could be made by tumor vaccines as well as adoptive transfer of tumor-specific T cells [13-15]. There has been a pressing need to identify immunological biomarkers predictive of therapy outcome that can be used to select patients and improve the response rates. From the presentation of Laszlo Radványi (MD Anderson Cancer Center, Houston, TX, USA), it was interesting to hear about functional aspects of Treg cells during high-dose IL-2 therapy of patients with melanoma and about changes in Treg cells associated with the treatment outcome. He found that $50 \%$ of human metastatic melanomas expressed inducible costimulator (ICOS)-ligand and provided ICOS costimulation through ICOS-ligand facilitating rapid expansion of an $\mathrm{ICOS}^{+}$Treg cell subset capable of IL-10 production and enhanced suppressive function. The ICOS ${ }^{+}$ and - Treg cell subsets showed characteristic changes in the course of the therapy. Eva Rajnavolgyi (University of Debrecen) spoke about DC subsets as the adjuvants for immunomodulatory vaccines. The inflammatory nature of CD1a $\mathrm{D}^{+} \mathrm{DC}$ was attributed to the expression and function of special intracellular sensors responsible for IFN- $\beta$

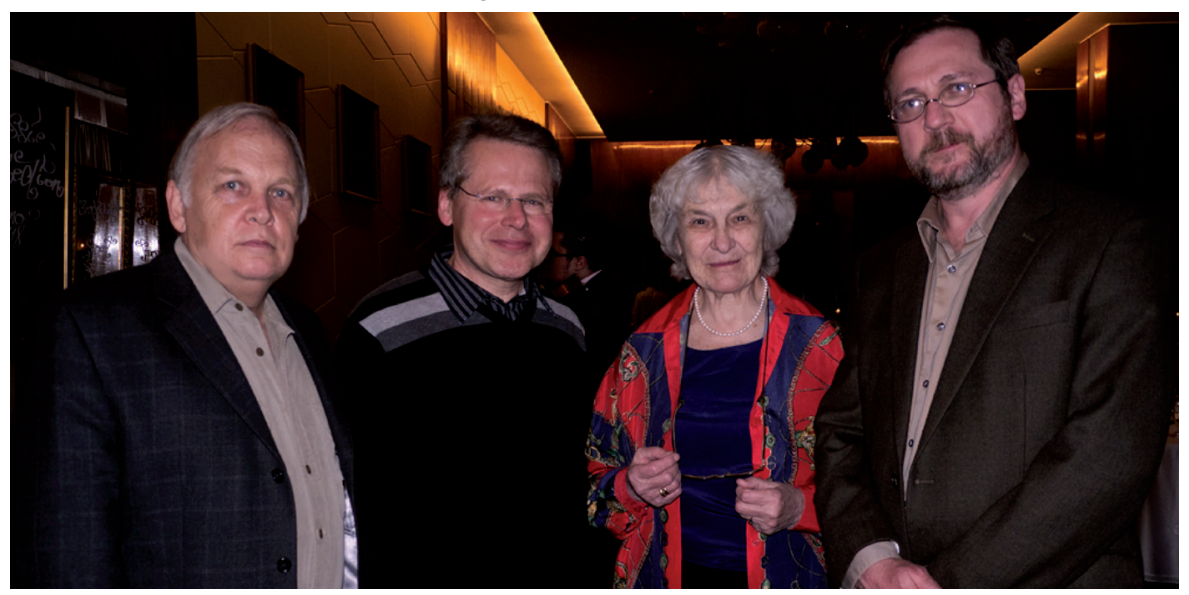

Figure 1. Main conference organizer and invited speakers. Anatoli M Malyguine, Viktor Umansky, Eva Klein and Michael R Shurin (conference chair; from left to right). 
production through the IRF3-TRIF pathway that ensures to create an inflammatory tissue environment.

Studies are further focused on the understanding of the relationship between immune and cancer cells and the context of the tumor microenvironment. Learning the basic mechanisms that can switch a chronic inflammatory process into an acute reaction with the power of destroying completely the triggering cause will be of help to guide the development of novel therapeutic strategies. Francesco Marincola $\mathrm{NIH}$, Bethesda, MD, USA) highlighted two molecular phenotypes being observed when cancers become established: one is characterized by the tumor microenvironment infiltrated by immune cells bearing transcriptional signatures consistent with a status of partial activation. Immune infiltration is considered to have a favorable prognostic or predictive association with the history of the disease or response to therapy. This overview on the common signatures of rejection with overlapping mechanisms leading to tumor rejection and other immune-mediated tissuespecific destructions (allograft rejection, graft-versus-host disease, acute clearance of pathogens and autoimmunity) gave a strong impression on the achievements in the field [12].

Identification of interferon (IFN) resistance gene signatures of human melanoma to improve patient selection for conventional therapy could be an example to emphasize the importance of these investigations. Jozsef Timar (Institute of Pathology, Semmelweis University, Budapest, Hungary) gave an essential information about the in vivo establishment of human melanoma IFN resistance gene signature consisting of 32 genes. The overwhelming majority of these genes $(78 \%)$ was found to be upregulated. Since cytokine resistance is connected to metastatic phenotype, a human melanoma spontaneous metastasis model was tested, where metastatic potential-related metastasis-initiating gene signature could be identified in metastatic versus nonmetastatic primary tumors and metastasis-maintenance gene signature could be obtained by comparing metastasis with the primary tumors.
There were only few people who presented studies on B cells at CITIM conference. Nevertheless the field is slowy accumulating relevant tumor immunological information. Everybody gladly greeted Eva Klein (Karolinska Institute, Stockholm, Sweden) in Hungary and learnt a lot from her previous pioneering and present novel studies on lymphokine modification of the expression of epstein-barr virus-encoded proteins in B lymphocytes. Galina Kaseko (Stephen Sanig Research Institute, Syndey, Australia) reported an efficient method of immortalizing rare B lymphocytes by using one-on-one electrical cell hybridization with their established human hematopoietic hybrid cell line as partner cells. The approach allows a fast establishment of unique B-cell-derived hybridoma bank for structural and functional analysis of natural tumor antibody repertoire. Small quantities of B cells can be found in tumors (tumor-infiltrating lymphocytes [TILs] such as TIL-B) and this and other methods, the efficient TIL-B antibody phage display, may help to understand the mechanisms underlying the natural antibodies responses to tumor antigens and to identify novel tumor associated antigens. This was already proved in a previous paper of Beatrix Kotlan (National Institute of Oncology, Budapest, Hungary) in relation to unique sialilated glycosphingolipides, GD3 gangliosides [16]. The presented results evidenced that the TIL-B approach is promising for the development of antitumor antibodies. The developed singlechain Fv immunoglobulin variable-region gene fragments of human origin were proved to be directed against specific disialolactosyl ceramides (GD3) and might be a tool for the early detection of unique glycosilated residues. The importance of natural antibodies was further emphasized in the lecture of Miri Blank from Yehuda Shoenfeld's group (Sheba Medical Center, Tel Aviv University, Israel) discussing their studies on intravenous immunoglobulin preparations in malignant conditions. intravenous immunoglobulin serves as an example of harnessing the innate immunity for treating incapacitating medical conditions, such as autoimmunity and cancer [17]. Paolo Ascierto's (Istituto Nazionale per lo Studio e la Cura dei Tumori 'Fondazione Pascale', Naples, Italy) 

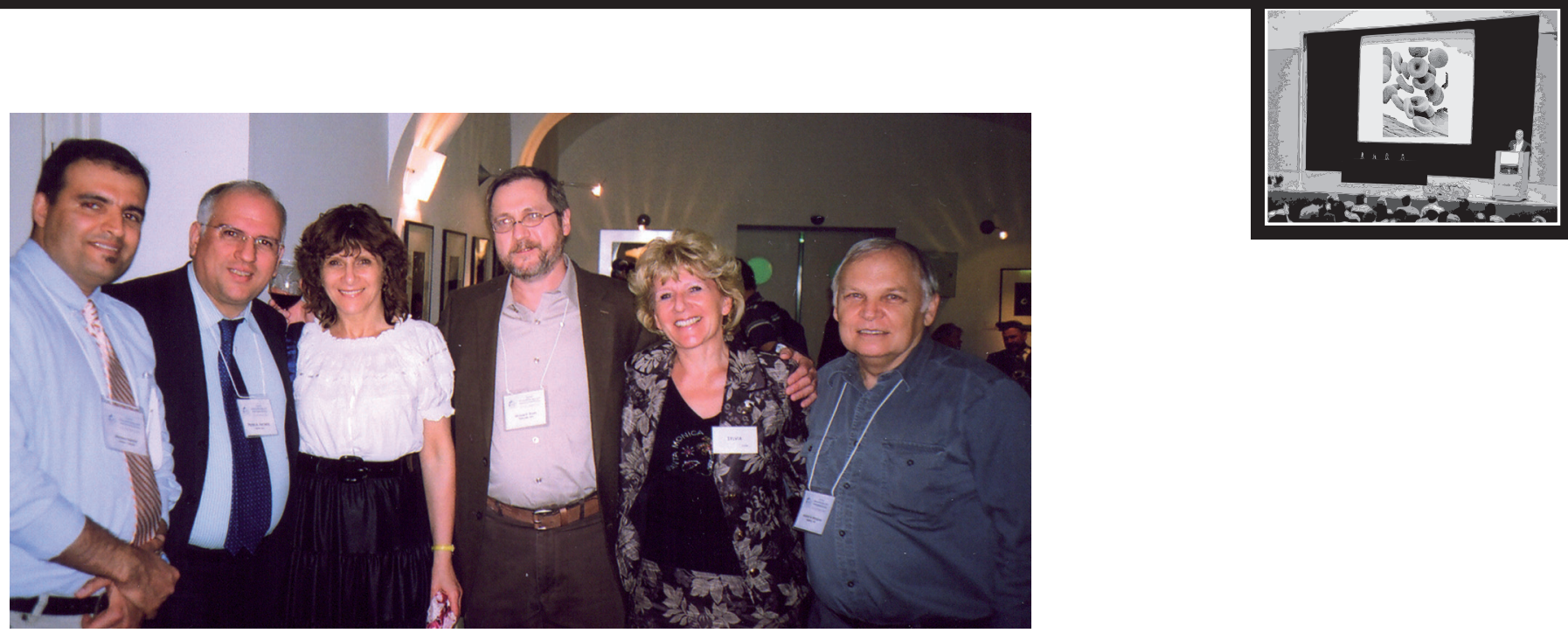

Figure 2. Welcome of Cancer Immunotherapy and Immunomonitoring Conference (CITIM) 2011: organizers and participants. SM Mansour Haeryfar, Paolo Ascierto,

Beatrix Kotlan, Michael Shurin, Sylvia Kotlan and Anatoli M Malyguine (from left to right).

presentation on immunomodulating antibodies gave a perfect view of the state of the art of targeted therapies [18] and of the different approaches to improve the efficacy of melanoma therapies. Monoclonal antibodies that block the CTL-associated antigen-4 (CTLA-4) potentiate the T-cell response against tumor; preliminary data showed their good tolerability and efficacy in the treatment of metastatic melanoma. Some other approaches (e.g., agonist antibodies directed to CD137 on antigenprimed T cells, interference with PD1 cell surface molecule or its ligand), are under investigation and used in clinical trials. As new class of drugs in cancer therapy, immunostimulatory monoclonal antibodies lead to the redefinition of tumor response criteria and potential combination therapies (immunotherapy, biotherapy and chemotherapy).

The lecture of Malcolm Brenner (Baylor College of Medicine, Houston, TX, USA) provided a fascinating picture of how improvements in cellular biology and the ability to manipulate gene expression in cells of the immune system have influenced the development of T-cell therapies. The presentation described how adoptively transferred $T$ cells can be used in the therapy of solid tumors and hematologic malignancy. Their clinical study of neuroblastomas confirmed the in vivo superiority of CAR-CTL over CAR-T cells and indicated a means by which T-cell therapy for cancer can be improved. Laurence JN Cooper (MD

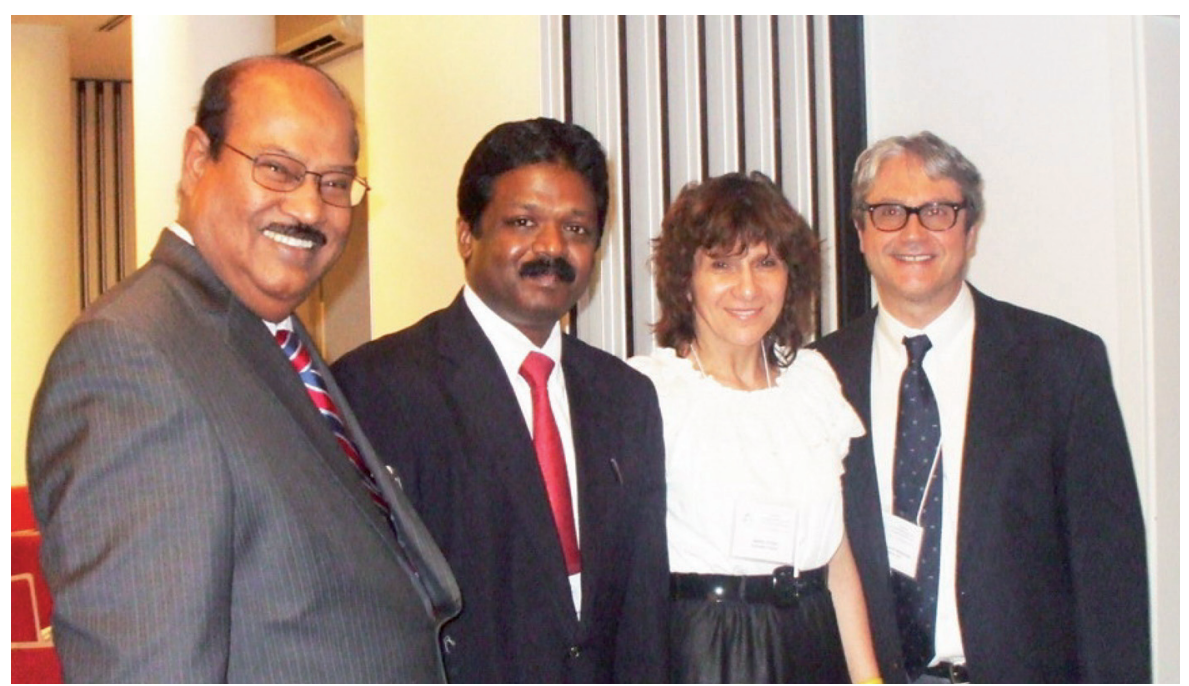

Figure 3. Welcome of Cancer Immunotherapy and Immunomonitoring Conference (CITIM) 2011: organizers and participants. Mepur H Ravindranath, Samuel JK Abraham, Beatrix Kotlan and Francesco M Marincola (from left to right). 
Anderson Cancer Center) presented a novel nonviral approach for the gene transfer of CARs into $T$ cells, the electrotransfer of 'Sleeping Beauty' (SB) transposon and transposases system. They developed the human application for improvement of this strategy. The SB system was used to express a transposon coding for a single-chain CAR redirecting T-cell specificity for a tumorassociated antigen, such as CD19. As further innovation, the DNA plasmids coding for the CAR (transposon) and hyperactive transposase (SB11) could be produced as a clinical grade material at many facilities [19]. Clinical trials could be initiated under the auspices of the US FDA to patients with aggressive $\mathrm{B}$ lymphoid malignancies.

Mepur Ravindranath (Terasaki Foundation Laboratory, CA, USA) presented their interesting findings on HLA class Ia and HLA-E antibodies in melanoma patients in response to autologous vaccine. The expression of HLA-E is augmented when the expression of MHC-Ia alleles is downregulated in tumor cells. Concomitant with the increase in antiHLA-E IgG, the patients also showed an increase in antibodies reacting to HLA-B and - $\mathrm{Cw}$ alleles. HLA-E and HLA-Ia reactivities of the sera could be inhibited by peptide sequences shared by HLA-E and the allo-HLA-B and - $\mathrm{Cw}$ alleles. Soluble HLA-E shed from the tumor cells in situ, may expose immunogenic cryptic domains that may elicit antibodies that react with HLA-E and HLA-Ia alleles that share the peptide sequences.

Joseph G Sinkovics (St Joseph Hospital's Cancer Institute, Moffitt Comprehensive Cancer Center, Department of Molecular Medicine, University of South Florida College of Medicine, FL, USA) interestingly summarized the processes of inflammatory carcinogenesis, in terms of 'The wild-type original and the tamed contemporary DNA' and how the molecular mediators promote carcinogenesis. To get more detailed view on that, we recommend his relevant book [20].

\section{Conclusion}

This international conference on immunotherapy, the first in Hungary, gave a platform for the presentation and discussion of a great variety of scientific results, already clinically introduced novelties 'on the way to clinical trials'. The future is going towards a combination of immunotherapy, biological therapy and chemotherapy [21]. The aim of advancing the understanding and application of immunotherapy helps to reach next milestones for better cancer cure rates.

\section{Take-home message}

The CITIM-2011 conference presentations and discussions gave strong evidence that we might look to a brighter future of the immunotherapeutic protocols as the therapeutic tools to fight against cancer (Figures 1-3). The extension of the valuable information about these new modalities in a translational form is a considerable asset for Central and Eastern European countries, from where scientists and clinicians could come to this meeting. This way, the novel 'scientific milestones' could reach out to farther 'geographic milestones' and will help a more broad group of patients suffering from cancer. Anticancer drug development has been going through a transition and now this transition is passing into clinical practice. Through better understanding of cancer biology, the therapies should be targeted to act specifically on cancer cells. Either a smaller or a larger number of cancer patients in our countries or all over the world will benefit from the novel immunotherapeutic strategies, the input effort into this conference was certainly not in vain. Additional information about this and future CITIM conference can be found at [101].

\section{Acknowledgements}

The authors would like to thank all International Organizing Committee Members, the Scientific Advisory Committee and the Hungarian Organizing committee for their important input into the meeting. The authors would also like to express their gratitude to Sylvia Kotlan for her outstanding organizational input in all aspects of conference arrangements that was absolutely essential for the success of CITIM-2011 conference. The authors would also like to express their thanks to Nora Kotlan for her helpful suggestions.

\section{Author disclosure}

The content of this publication does not necessarily reflect the views or policies of the Department of Health and Human Services, nor does mention of trade names, commercial products, or organizations imply endorsement by the US Government. 


\section{Financial \& competing interests disclosure}

The authors acknowledge the generous support of the conference sponsors: Cellular Technology Ltd, GTCbio, Nodality Inc, Roche (Hungary) Ltd, Cancer Immunology and Immunotherapy, Journal of Immunotoxicology, Miltenyi Biotech GmbH, Epiontis GmbH, eBioscience Inc, Biolegend, Fulbright Program (Hungarian-American Fulbright Commission, Ceragem. The authors thank the Department of Pathology, University of Pittsburgh Medical Center, Baylor College of Medicine, Texas Children's Cancer Center, Cancer Immunology and Immunotherapy, Vitachemix LLC for their help. The authors acknowledge Harry J Lloyd Charitable Trust Melanoma Research Grant for the possibility of two collaborating professors' attendance. This project has been funded in whole

\section{Bibliography}

1 Wang E, Panelli MC, Monsurró V, Marincola FM. A global approach to tumor immunology. Cell. Mol. Immunol. 1(4), 256-265 (2004).

2 Bedognetti D, Wang E, Sertoli MR, Marincola FM. Gene-expression profiling in vaccine therapy and immunotherapy of cancer. Expert Rev. Vaccines 9(6), 555-565 (2010).

3 Gajewsky TF. Transcriptional profiling of melanoma as a potential predictive biomarker for response to immunotherapy. In: Immunologic Signatures of Rejection. Marincola FM, Wang E (Eds). Springer, NY, USA 229-238 (2011).

4 Singh H, Figliola MJ, Dawson MJ et al. Reprogramming CD19-specific T cells with IL-21 signaling can improve adoptive Immunotherapy of B-lineage malignancies. Cancer Res. 71(10), 3516-3527 (2011).

5 Malyguine A, Umansky V, Kotlan B, Aptsiauri N, Shurin MR. Cancer immunotherapy and immunomonitoring: moving forward. J. Immunotoxicol. (In Press) (2011).

6 Umansky V, Malyguine A, Kotlan B, Aptsiauri N, ShurinMR. Cancer immunotherapy immunomonitoring (CITIM) meeting held in Budapest, 2-5 May. Cancer Immunol. Immunother. (In Press) (2011).

7 Cavallo F, Giovanni CD, Nanni P, Forni G, Lolini PL. The immune hallmarks of cancer. Cancer Immunol. Immonother. 60, 319-326 (2011). or in part with federal funds from the National Cancer Institute, NIH, under Contract No. HHSN261200800001E. The project was also funded by Dr Mildred Scheel Foundation for Cancer Research grant 108992 (to Viktor Umansky). The authors would also like to thank CERAGEM ('Healing your life') for generously sponsoring a great farewell dinner in Hungarian style in the famous 100-year-old Gellert Hotel on the Danube river bank - as a cheerful nice closing event - the participants might still remember with the Budapest by night view. The authors have no other relevant affiliations or financial involvement with any organization or entity with a financial interest in or financial conflict with the subject matter or materials discussed in the manuscript apart from those disclosed.

No writing assistance was utilized in the production of this manuscript.

8 Balwit JM, Hwu P, Urba WJ, Marincola FM. The iSBTC/SITC primer on tumor immunology and biological therapy of cancer. a summary of the (2010) program. J. Transl. Med. 9, 18-32 (2011).

9 Ferrantini MF, Capone I, Marincola FM, Parmiani G, Belardeli F. International Meeting 'Immunotherapy of Cancer: Challenges and Needs'. Cancer Immunol. Immunother. 56, 581-585 (2007).

10 Butterfiel LH, Disis ML, Fox BA et al. A systematic approach to biomarker discovery. Preamble to 'the iSBTc-FDA Taskforce on Immunotherapy Biomarkers'. J. Transl. Med. 6, 81-91 (2008).

11 Mantovani A, Garlanda C, Allevana P, Sica A, Locati M. The Ying Yang of cancer related inflammation. In: Immunologic Signatures of Rejection. FM Marincola, E Wang (Eds). Springer NY, USA 11-16 (2011).

12 Wang E, Marincola FM. Immune mediated tumor rejection. In: Immunologic Signatures of Rejection. FM Marincola, E Wang (Eds). Springer, NY, USA 281-304 (2011).

13 Kershaw MH., Westwood JA, Parker LL et al. A Phase I study on adoptive immunotherapy using gene-modified $\mathrm{T}$ cells for ovarian Cancer. Clin. Cancer Res. 12(20), 6106-6015 (2006).

14 Rosenberg SA, Dudley ME. Adoptive cell therapy for the treatment of patients with metastatic melanoma. Curr. Opin Immunol. 21(2), 233-240 (2009).

15 Engell-Noerregaard L, Hansen MH, Straten P, Svane IM. Review of clinical studies on Dendritic cell based vaccination of patients with malignant melanoma. assessment of correlation between clinical response and vaccine parameters. Cancer Immunol. Immunother. 5(1), 1-14 (2009).

16 Kotlan B, Simsa P, Teillaud JL et al. Novel ganglioside antigen identified by $\mathrm{B}$ cells in human medullary breast carcinomas: the proof of principle concerning the infiltrating B lymphocytes. J. Immunol. 175(4), 2278-2285 (2005).

17 Shoenfeld Y, Krause I. IVIG for autoimmune, fibrosis and malignant conditions: our experience with 200 patients. J. Clin. Immunol. 24(2), 107-114 (2004).

18 Begley J, Ribas A. Targeted therapies to improve tumor immunotherapy. Clin. Cancer Res. 14(14), 4385-4391 (2008).

19 Jin Z, Maitit S, Huls H et al. The hyperactive Sleeping Beauty transposase SB100X improves the genetic modification of $\mathrm{T}$ cells to express a chimeric antigen receptor. Gene Ther. doi.10.1038/gt.2011.40 (2011) (Epub ahead of print).

20 Sinkovics JG. "Up-dating the monograph." [Corrected] Cytolytic immune lymphocytes, in the armamentarium of the human host. Acta Microbiol. Immunol. Hung. 55(4), 371-382 (2008).

21 Ascierto PA, Marincola FM. Combination therapy: the next opportunity and challenge of medicine. J. Transl. Med. 9(1), 115 (2011).

\section{Website}

101 CITIM 2011 Budapest conference homepage www.CancerITIM.org 